\title{
Unsteady boundary layer flow of a Casson fluid past an oscillating vertical plate with constant wall temperature
}

\author{
Asma Khalid ${ }^{1,2}$, Ilyas Khan ${ }^{3}$, Sharidan Shafie ${ }^{2, *}$ \\ ${ }^{1}$ Department of Mathematics, SBK Women's University, Quetta 87300, Pakistan. \\ ${ }^{2}$ Department of Mathematical Sciences, Faculty of Science, Universiti Teknologi Malaysia 81310 UTM Skudai \\ ${ }^{3}$ College of Engineering Majmaah University, Majmaah 11952, Saudi Arabia. \\ *Corresponding Author: sharidan@utm.com
}

Article history :

Received 11 September 2014

Accepted 25 February 2015

\section{GRAPHICAL ABSTRACT}

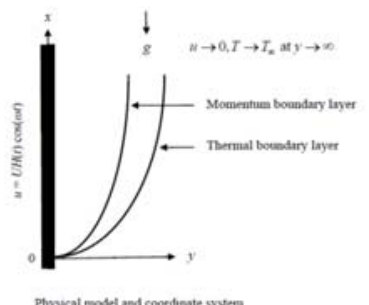

\begin{abstract}
The unsteady boundary layer flow is presented for non-Newtonian fluid flow past an oscillating vertical plate with constant wall temperature. The Casson fluid model is used to distinguish the non-Newtonian fluid behavior. The governing partial differential equations corresponding to the momentum and energy equations are transformed into dimensionless forms, by using suitable transformations. Laplace transform method is used to find the exact solutions of these equations. The expressions for shear stress in terms of skin friction and the rate of heat transfer in terms of Nusselt number are also obtained. Numerical results of velocity and temperature profiles with various values of embedded flow parameters are shown graphically and their effects are discussed in detail.
\end{abstract}

Keywords: Casson fluid, Boundary layer flow, Oscillating vertical plate, Similarity transformations

C 2015 Penerbit UTM Press. All rights reserved http://dx.doi.org/10.11113/mjfas.v11n1.360

\section{INTRODUCTION}

It is a well know fact that many complex fluids such as blood, soap, clay coating, certain oils and greases, elastomers, suspensions and many emulsions are noteworthy due to their various applications in industries. But unfortunately Navier Stokes equations are no more valid to describe them. Such fluids are known as non-Newtonian fluids. These fluids are described by a non-linear relationship between the stress and the rate of strain. Therefore, mostly it is not easy to find their solution, more specifically exact solutions same like for Newtonian fluids. Newtonian fluids on the other hand are relative easy and convenient for exact solutions [1-5].

The interest of researchers to study nonNewtonian fluid is motivated because of their extensive applications in diverse areas of biorheology, geophysics, chemical and petroleum industries. In view of these particular applications, the study and understanding of non-Newtonian fluids have now become an increasingly appealing topic of current research in this field. The most difficult task in studying non-Newtonian fluids is, that they cannot be described by using a single mathematical model like Newtonian fluids. Therefore, for each non-Newtonian fluid there is a separate model of constitutive equations. Amongst them there is one non-Newtonian fluid model which is recently quite famous called Casson fluid model. Basically this fluid model was introduced by Casson in 1959 to study the prediction of the flow behavior of pigment-oil suspensions [6]. After the seminal work of Casson, several authors including Hayat et al. [7], Mukhopadhyay [8], Bhattacharyya [9], Mukhopadhyay et al. [10] and Pramanik [11] have used Casson fluid model in their respective problems. However most of them analyzed their problems using either approximate or numerical methods. Some famous examples of Casson fluid include jelly, tomato sauce, honey, soup and concentrated fruit juices amongst others.

Exact solutions of Casson fluid are scarce. They are even more rare when the flow of Casson fluid is needed to study in the presence of free convection. Due to this reason we are motivated to study this problem. More exactly, in this article we have considered the unsteady free convection flow of Casson fluid past an oscillating vertical plate which obeys the constant wall temperature condition. Exact solutions are obtained by using the Laplace transform technique. Analytical results for skin-friction and Nusselt number are provided. Graphical results are presented and discussed for various physical parameters. Exact solutions obtained in this paper can be used as a bench mark for validation of other solutions obtained via approximate or numerical schemes.

\section{FORMULATION OF THE PROBLEM}

We consider Casson fluid past an infinite vertical flat plate situated at the flow being confined to $y>0$, where $y$ is the coordinate measured in the normal direction to the surface. It is assumed that at the initial moment $t=0$, both the plate and fluid are at rest with constant temperature $T_{\infty}$. At time $t=0^{+}$the plate begins to oscillate in its plane $(y=0)$ according to 
$\mathbf{V}=U H(t) \cos (\omega t) \mathbf{i}$,

where the constant $U$ is the amplitude of the plate oscillations, $H(t)$ is the unit step function, $\mathbf{i}$ is the unit vector in the vertical flow direction and $\omega$ is the frequency of oscillation of the plate. At the same time, the plate temperature is raised to $T_{w}$ which is thereafter maintained constant. The corresponding equations for momentum and energy under the above assumptions are given by

$\rho \frac{\partial u}{\partial t}=\mu\left(1+\frac{1}{\gamma}\right) \frac{\partial^{2} u}{\partial y^{2}}+\rho g \beta\left(T-T_{\infty}\right)$,

$\rho c_{p} \frac{\partial T}{\partial t}=k \frac{\partial^{2} T}{\partial y^{2}}$

together with initial and boundary conditions

$$
\begin{gathered}
t<0: u=0, T=T_{\infty} \text { for all } y>0, \\
t \geq 0: u=U H(t) \cos (\omega t), T=T_{w} \text { at } y=0 \\
u \rightarrow 0, T \rightarrow T_{\infty} \text { as } y \rightarrow \infty .
\end{gathered}
$$

We introduce the following dimensionless variables

$$
\begin{aligned}
& u^{*}=\frac{u}{U}, y^{*}=\frac{U}{v} y, t^{*}=\frac{U^{2}}{v} t, \\
& \theta=\frac{T-T_{\infty}}{T_{w}-T_{\infty}}, \omega^{*}=\frac{\omega v}{U^{2}}, \tau^{*}=\frac{\tau}{\rho u^{2}},
\end{aligned}
$$

into equations (2-4), and we get (* symbols are dropped for simplicity)

$$
\begin{aligned}
& \frac{\partial u}{\partial t}=\left(1+\frac{1}{\gamma}\right) \frac{\partial^{2} u}{\partial y^{2}}+G r \theta, \\
& \operatorname{Pr} \frac{\partial \theta}{\partial t}=\frac{\partial^{2} \theta}{\partial y^{2}}
\end{aligned}
$$

with associated initial and boundary conditions

$$
\begin{gathered}
t<0: u=0, \theta=0 \text { for all } y>0, \\
t \geq 0: u=H(t) \cos (\omega t), \theta=1 \text { at } y=0, \\
u \rightarrow 0, \theta \rightarrow 0 \text { as } y \rightarrow \infty,
\end{gathered}
$$

where

$$
\operatorname{Pr}=\frac{\mu c p}{k} \text {, and } G r=\frac{v g \beta\left(T_{w}-T_{\infty}\right)}{U^{3}},
$$

are the Prandtl and Grashof numbers.

\section{SOLUTION OF THE PROBLEM}

Applying Laplace transforms to equations (6) and (7), using initial and boundary conditions (8), we get the following solutions in the transformed plane

$$
\begin{aligned}
\bar{\theta}(y, q) & =\frac{1}{q} e^{-y \sqrt{\operatorname{Pr} q}}, \\
\bar{u}(y, q)= & \frac{q}{q^{2}+\omega^{2}} e^{-y \sqrt{a q}}+\frac{b}{q^{2}} e^{-y \sqrt{a q}} \\
& +a \frac{b}{q^{2}} e^{-y \sqrt{\operatorname{Pr} q}} .
\end{aligned}
$$

The inverse Laplace transforms of equations (9) and (10) are obtained as follows:

$$
\begin{aligned}
& \theta(y, t)=\operatorname{erf} c\left(\frac{y}{2} \sqrt{\frac{\operatorname{Pr}}{t}}\right), \\
& u(y, t)=u_{m e}(y, t)+u_{t h}(y, t), \\
& u_{m e}(y, t)=\frac{H(t)}{4} e^{-i \omega t}\left[\begin{array}{l}
e^{-y \sqrt{-i \omega a}} \operatorname{erf} c\left(\frac{y}{2} \sqrt{\frac{a}{t}}-\sqrt{-i \omega t}\right) \\
+e^{y \sqrt{-i \omega a}} \operatorname{erf} c\left(\frac{y}{2} \sqrt{\frac{a}{t}}+\sqrt{-i \omega t}\right)
\end{array}\right] \\
& +\frac{H(t)}{4} e^{i \omega t}\left[\begin{array}{c}
e^{-y \sqrt{i \omega a}} \operatorname{erf} c\left(\frac{y}{2} \sqrt{\frac{a}{t}}-\sqrt{i \omega t}\right) \\
+e^{y \sqrt{i \omega a}} \operatorname{erf} c\left(\frac{y}{2} \sqrt{\frac{a}{t}}+\sqrt{+i \omega t}\right)
\end{array}\right],
\end{aligned}
$$

$$
\begin{aligned}
& u_{t h}(y, t)=a b\left[\begin{array}{l}
\left(t+\frac{a y^{2}}{2}\right) \operatorname{erf} c\left(\frac{y}{2} \sqrt{\frac{a}{t}}\right) \\
-y \sqrt{a} \sqrt{\frac{t}{\pi}} e^{-\frac{a y^{2}}{4 t}}
\end{array}\right] \\
& +a b\left[\begin{array}{l}
\left(t+\frac{\operatorname{Pr} y^{2}}{2}\right) \operatorname{erf} c\left(\frac{y}{2} \sqrt{\frac{\operatorname{Pr}}{t}}\right) \\
-y \sqrt{\operatorname{Pr}} \sqrt{\frac{t}{\pi}} e^{-\frac{\operatorname{Pr} y^{2}}{4 t}}
\end{array}\right],
\end{aligned}
$$

with

$$
a=\frac{\gamma}{1+\gamma}, b=\frac{G r}{\operatorname{Pr}-1},
$$

where, $u_{m e}(y, t)$ denotes the mechanical part and $u_{t h}(y, t)$ is the corresponding thermal part.

Note that the above solution (12) for velocity is only valid for $\operatorname{Pr} \neq 1$. Moreover the solution for $\operatorname{Pr}=1$ can be easily obtained by putting $\operatorname{Pr}=1$ into equation (7), and follow a similar procedure as discussed above, we obtain 


$$
\begin{aligned}
& u_{c}(y, t)=\frac{H(t)}{4} e^{-i \omega t}\left[\begin{array}{l}
e^{-y \sqrt{-i \omega a}} \operatorname{erf} c\left(\frac{y}{2} \sqrt{\frac{a}{t}}-\sqrt{-i \omega t}\right) \\
+e^{y \sqrt{-i \omega a}} \operatorname{erf} c\left(\frac{y}{2} \sqrt{\frac{a}{t}}+\sqrt{-i \omega t}\right)
\end{array}\right] \\
& +\frac{H(t)}{4} e^{i \omega t}\left[\begin{array}{l}
e^{-y \sqrt{i \omega a}} \operatorname{erf} c\left(\frac{y}{2} \sqrt{\frac{a}{t}}-\sqrt{i \omega t}\right) \\
+e^{y \sqrt{i \omega a}} \operatorname{erf} c\left(\frac{y}{2} \sqrt{\frac{a}{t}}+\sqrt{+i \omega t}\right)
\end{array}\right] \\
& +\frac{a \operatorname{Gr} y}{2}\left[2 \sqrt{\frac{t}{\pi}} e^{-\frac{y^{2}}{4 t}}-y \operatorname{erf} c\left(\frac{y}{2 \sqrt{t}}\right)\right] \text {. }
\end{aligned}
$$

The expression for skin-friction and Nusselt number are

$$
\begin{aligned}
\tau= & -\left.\mu\left(1+\frac{1}{\gamma}\right) \frac{\partial u}{\partial y}\right|_{y=0}, \\
\tau= & \frac{1}{2 a_{1} \sqrt{\pi}}\left[e ^ { - i t \omega } \left[2 e ^ { i t \omega } \left\{H \sqrt{\frac{a_{1}}{t}}+\sqrt{a_{1}} b \sqrt{t}\right.\right.\right. \\
& \left.\left.-a_{1} b \sqrt{\operatorname{Pr}} \sqrt{t}+b \sqrt{\frac{a_{1}}{t}} t+a_{1} b \sqrt{\frac{\operatorname{Pr}}{t}} t\right)\right\} \\
& +H \sqrt{\pi}\left\{\sqrt{-i a_{1} \omega} \operatorname{erf}(\sqrt{-i t \omega})\right. \\
& \left.\left.\left.+e^{2 i t \omega} \sqrt{i a_{1} \omega} \operatorname{erf}(\sqrt{i t \omega})\right\}\right]\right], \\
& \mathrm{Nu}=-\left.\frac{v}{U_{0}\left(T-T_{\infty}\right)} \frac{\partial T^{*}}{\partial y^{*}}\right|_{y^{*}=0}=\sqrt{\frac{\operatorname{Pr}}{\pi t}} .
\end{aligned}
$$

\section{LIMITING CASES}

The following solutions from the literature appear as the limiting cases of our general solutions.

(i). By taking $\gamma \rightarrow \infty$ into equation (14), the corresponding solutions for viscous fluid can be obtained as a special case:

(ii).

$$
\begin{aligned}
& u_{c}(y, t)=\frac{H(t)}{4} e^{-i \omega t}\left[\begin{array}{l}
e^{-y \sqrt{-i \omega}} \operatorname{erf} c\left(\frac{y}{2} \sqrt{\frac{1}{t}}-\sqrt{-i \omega t}\right) \\
+e^{y \sqrt{-i \omega}} \operatorname{erf} c\left(\frac{y}{2} \sqrt{\frac{1}{t}}+\sqrt{-i \omega t}\right)
\end{array}\right] \\
& +\frac{H(t)}{4} e^{i \omega t}\left[\begin{array}{c}
e^{-y \sqrt{i \omega}} \operatorname{erf} c\left(\frac{y}{2} \sqrt{\frac{1}{t}}-\sqrt{i \omega t}\right) \\
+e^{y \sqrt{i \omega}} \operatorname{erf} c\left(\frac{y}{2} \sqrt{\frac{1}{t}}+\sqrt{+i \omega t}\right)
\end{array}\right] \\
& +b\left[\left(t+\frac{y^{2}}{2}\right) \operatorname{erf} c\left(\frac{y}{2} \sqrt{\frac{1}{t}}\right)-y \sqrt{\frac{t}{\pi}} e^{-\frac{y^{2}}{4 t}}\right] \\
& +b\left[\left(t+\frac{\operatorname{Pr} y^{2}}{2}\right) \operatorname{erf} c\left(\frac{y}{2} \sqrt{\frac{\operatorname{Pr}}{t}}\right)-y \sqrt{\operatorname{Pr}} \sqrt{\frac{t}{\pi}} e^{-\frac{\operatorname{Pr} y^{2}}{4 t}}\right] .
\end{aligned}
$$

(iii). By taking $\omega=0$, which corresponds to impulsive motion of the plate, then equations (12) yield

$$
\begin{aligned}
u_{c}(y, t)= & H(t)\left[\operatorname{erf} c\left(\frac{y}{2} \sqrt{\frac{a}{t}}\right)\right] \\
& +a b\left[\left(t+\frac{a y^{2}}{2}\right) \operatorname{erf} c\left(\frac{y}{2} \sqrt{\frac{a}{t}}\right)-y \sqrt{a} \sqrt{\frac{t}{\pi}} e^{-\frac{a y^{2}}{4 t}}\right] \\
& +a b\left[\left(t+\frac{\operatorname{Pr} y^{2}}{2}\right) \operatorname{erf} c\left(\frac{y}{2} \sqrt{\frac{\operatorname{Pr}}{t}}\right)-y \sqrt{\operatorname{Pr}} \sqrt{\frac{t}{\pi}} e^{-\frac{\operatorname{Pr} y^{2}}{4 t}}\right]
\end{aligned}
$$

equation (20) describe the corresponding solution of Stokes' first problem for Casson fluid. It is important to note that exact solution of Stokes' first problem for Casson fluid (20) is also not reported in the literature and hence is new.

(iv). In this last case, we assume that the flow is induced only due to bounding plate and the corresponding buoyancy forces are zero equivalently it shows the absence of free convection $(G r=0)$ due to the differences in temperature gradient. This shows that the thermal parts of velocity in equation (14) is zero. Hence the flow is only governed by the corresponding mechanical part given by

$$
\begin{aligned}
& u_{m e}(y, t)=\frac{H(t)}{4} e^{-i \omega t}\left[\begin{array}{l}
e^{-y \sqrt{-i \omega a}} \operatorname{erf} c\left(\frac{y}{2} \sqrt{\frac{a}{t}}-\sqrt{-i \omega t}\right) \\
+e^{y \sqrt{-i \omega a}} \operatorname{erf} c\left(\frac{y}{2} \sqrt{\frac{a}{t}}+\sqrt{-i \omega t}\right)
\end{array}\right] \\
& +\frac{H(t)}{4} e^{i \omega t}\left[\begin{array}{l}
e^{-y \sqrt{i \omega a}} \operatorname{erf} c\left(\frac{y}{2} \sqrt{\frac{a}{t}}-\sqrt{i \omega t}\right) \\
+e^{y \sqrt{i \omega a}} \operatorname{erf} c\left(\frac{y}{2} \sqrt{\frac{a}{t}}+\sqrt{+i \omega t}\right)
\end{array}\right] .
\end{aligned}
$$

Note that equation (21) is identical (when $\gamma \rightarrow \infty$ ) to that obtained by Fetecau et al. [9], see equation (8). This fact is also shown in figure 8 .

\section{RESULTS AND DISCUSSION}

In this section, the obtained exact solutions are studied numerically in order to determine the effects of Prandtl number $\mathrm{Pr}$, Grashof number $G r$, Casson parameter $\gamma$ and phase angle $\omega t$. It is observed from figure 1 that velocity decreases with increasing $\mathrm{Pr}$. Figure 2 elaborates that the velocity increases with increasing $\mathrm{Gr}$. Figure 3 shows influence of Casson parameter. It is found that velocity decreases with increasing values of $\gamma$. It is further observed from this figure that when the Casson parameter $\gamma$ is large enough i.e. $\gamma \rightarrow \infty$, the non-Newtonian behavior disappear and the fluid purely behaves like a Newtonian fluid. Thus, the velocity boundary layer thickness for Casson fluid is larger than the Newtonian fluid. It occurs because of plasticity of Casson fluid. Figure 4 shows the 
oscillatory behavior of velocity for different values of $\omega t$. This figure can easily help us to check the accuracy of our results. We can see that for these values of $\omega t$, the velocity shows its values either 1,0 or -1 which are identical with the imposed boundary conditions of velocity in equation (9). It is depicted from figure 5 that, the temperature profile decrease with increasing values of Prandtl number $\mathrm{Pr}$. It is observed that the thermal boundary layer thickness is maximum near the plate and decreases with increasing distance from the leading edge and finally approaches to zero. It is also noticed from this figure that the magnitude of temperature is greater for air compare to electrolytic solution and water.

For the sake of correctness and verification, we have compared our results with those of Fetecau et al. [9]. This comparison is shown in figure 6. It is found that our limiting solution (21) is identical to equation (8) obtained by Fetecau et al. [1]. This confirms the accuracy of our obtained result.

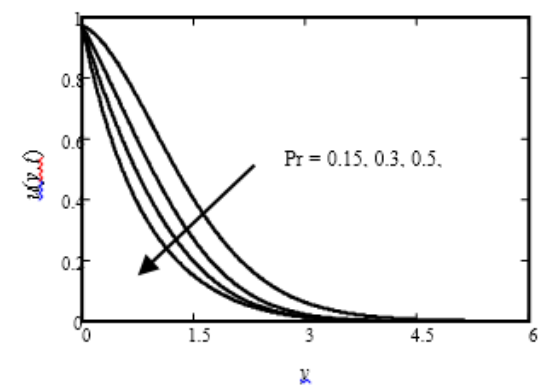

Figure 1: Profiles of velocity for different values of $\mathrm{Pr}$, when $\omega=\frac{\pi}{4}, t=0.2$ and $G r=3$.

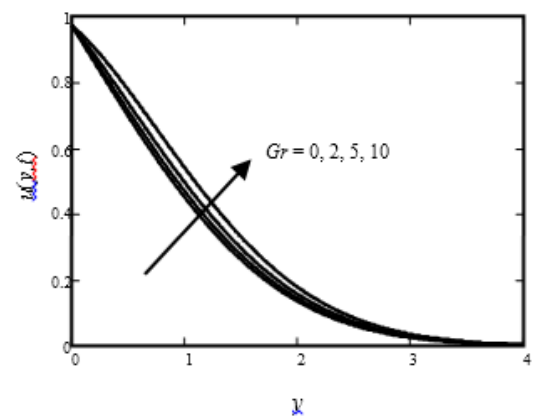

Figure 2: Profiles of velocity for different values of $G r$, when $\operatorname{Pr}=0.3, \gamma=0.6, t=0.3$ and $\omega=\frac{\pi}{4}$.

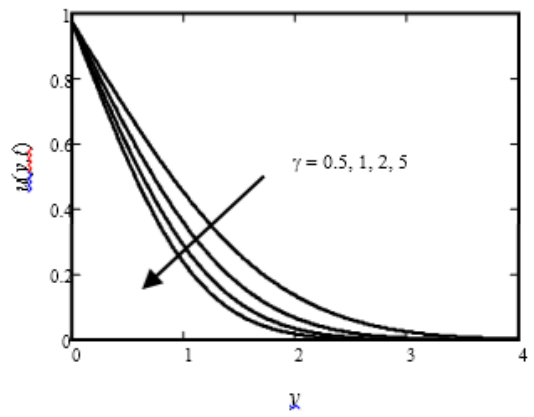

Figure 3: Profiles of velocity for different values of $\gamma$, where

$$
\operatorname{Pr}=0.3, G r=0, t=0.3 \text { and } \omega=\frac{\pi}{4} \text {. }
$$

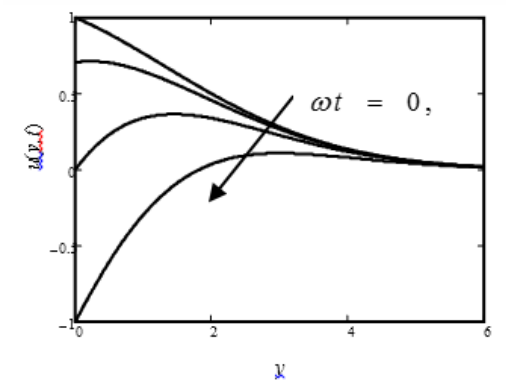

Figure 4: Profiles of velocity for different values of $\omega t$,

when $G r=3, \operatorname{Pr}=0.3, t=1$ and $\gamma=0.5$.

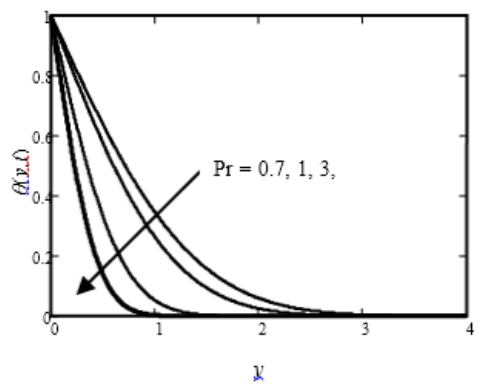

Figure 5: Profiles of temperature for different values of $\mathrm{P} \mathrm{r}$, when $t=0.4$.

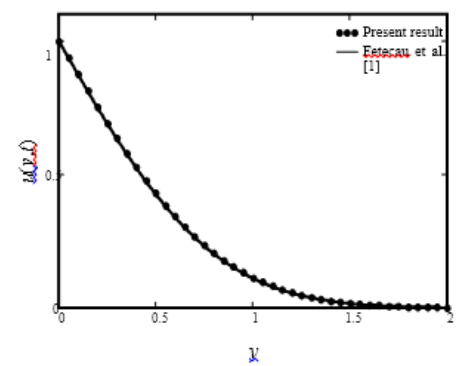

Figure 6: Comparison of the present results [see equation (21), when $\gamma \rightarrow \infty$ ] with those obtained by Fetecau et al. [1], [see equation (8)] whe $t=0.2, \omega=0, a=1, U=1$ and $v=1$.

\section{CONCLUSION}

Exact solutions for unsteady free convection flow of Casson fluid past an oscillating vertical plate with constant wall temperature are obtained. The Laplace transform technique is used for solution. Plots are prepared for velocity and temperature and discussed in details. It is found that the Casson fluid parameter have decreasing effect on velocity.

\section{ACKNOWLEDGEMENT}

The authors would like to acknowledge the SBKWU (HEC) Pakistan and MOHE for the financial support.

\section{References}

[1] Corina Fetecau,; Vieru, D.; Fetecau, C. Int. J. Non-linear Mech. 451-457 (2008) 43. 
[2] Erdogan, M. E. Int. J. Non-linear Mech. 1-6 (2000) 35.

[3] Mehmood, A.; Ali, A. Rom. J. Phys. 85-91 (2007) 52.

[4] Makinde, O. D.; Mhone, P.Y. Rom. J. Phys. 931-938 (2005) 50.

[5] A. Hussanan, A.; Anwar, M. I.; Ali, F. Khan, I.; Shafie, S. Heat Transfer Res. 119-135 (2014) 45.

[6] Casson, N. Pergamon, New York, 84-102 (1959).

[7] Hayat, T.; Awais M.; Sajid, M. Int. J. Mod. Phys. B. 2863-78 (2011) 25.
[8] Mukhopadhyay, S. Chin. Phys. B. Artic. ID 114702, (2013) 22.

[9] Bhattacharyya, K. (FHMT). Artic. ID 023003 (2013) 4.

[10] S. Mukhopadhyay, S.; De Rajan, P.; Bhattacharyya, K. Ain Shams Eng. J. 933-938 (2013) 4.

[11] Pramanik, S. Ain Shams Eng. J. 205-212, (2014) 5. 